\title{
Optimization and apoptosis induction by RNAi with UTMD technology in vitro
}

\author{
ZHI-YI CHEN $^{1 *}$, KUN LIANG $^{2}$, XIU-JIE SHENG ${ }^{2 *}$, BING SI-TU $^{3 *}$, XIAO-FANG SUN ${ }^{2}$, JIAN-QIAO LIU ${ }^{2}$, \\ RI-XIANG QIU ${ }^{3}$, HUA ZHANG ${ }^{1}$, YUE-WEI LI ${ }^{1}, X$ XIN-XIN ZHOU ${ }^{1}$ and JIANG-XIU YU ${ }^{1}$ \\ Departments of ${ }^{1}$ Medical Ultrasound, ${ }^{2}$ Obstetrics and Gynecology, and ${ }^{3}$ Pharmacy, \\ The Third Affiliated Hospital of Guangzhou Medical University, Guangzhou 510150, P.R. China
}

Received November 3, 2011; Accepted December 14, 2011

DOI: $10.3892 / \mathrm{ol} .2012 .610$

\begin{abstract}
Apoptosis induction by short hairpin RNA (shRNA) expression vectors may be an efficient and promising strategy for cancer gene therapy. Ultrasound-targeted microbubble destruction (UTMD) is an appealing technique; however, there few data are available to demonstrate the feasibility and to optimize the methodology for this technology. The aim of this study was to optimize this technique and to elucidate the effects on gene inhibition and apoptosis induction in vitro. Human cervical cancer cell lines were obtained and cultured. shRNA vectors were constructed, and the UTMD technique was examined to determine whether or not it was suitable for shRNA transfection into cells. Cells were then examined using flow cytometry. The results revealed that the optimal irradiation parameters obtained higher transfection efficiency and did not affect the integrity of plasmid DNA. We concluded that survivin downregulation with shRNA expression vectors, mediated by the optimal UTMD parameters, markedly induced cell apoptosis and caused cell cycle arrest, laying a foundation for further investigation of this cancer therapy.
\end{abstract}

\section{Introduction}

Apoptosis is a physiologically essential mechanism of the cell and is important in the development and progression of tumors. Regulated inhibition of apoptosis preserves normal homeostasis and tissue and organ morphogenesis. The clear strategy for cancer therapy is to target the lesions that suppress apoptosis in tumor cells (1). Diverse molecular mechanisms are involved in the induction of apoptosis $(2,3)$. Survivin, the smallest member of the mammalian inhibitors of the apoptosis protein (IAP) family, is upregulated in various malignancies

Correspondence to: Dr Zhi-Yi Chen, Department of Medical Ultrasound, The Third Affiliated Hospital of Guangzhou Medical University, DuoBao Road 63, Guangzhou 510150, P.R. China

E-mail: winchen@vip.126.com

*Contributed equally

Key words: ultrasound, microbubble, gene delivery, methodology, RNA interference, apoptosis, cancer to protect cells from apoptosis (4). Overexpression of survivin in cancer overcomes the apoptotic checkpoint and favors the aberrant progression through mitosis (5).

Gene silencing by RNA interference (RNAi) using synthetic small interfering RNA (siRNA) or expressed stem-loop RNA [short-hairpin RNA (shRNA) or artificial microRNA (miRNA)] has been shown to be effective against survivin in vitro and in vivo (6-8). However, due to the short half-life and low in vivo transfection efficiency of synthetic siRNA, the application of shRNA expression vectors, under the direction of RNA polymerase III promoters such as U6 and H1, may be a powerful tool for anticancer therapy $(9,10)$. shRNA was significantly more potent than siRNA at mediating knockdown, and the difference resulted from the less efficient delivery of siRNA to the cytosol compared with shRNA delivery to the nucleus (11). Moreover, shRNA was more effective than the artificial miRNA in mediating gene silencing, independently of the target sequence and experimental setting (12).

However, the utilization of shRNA expression vectors has been limited by the inefficient delivery technique, particularly in vivo (13). Currently, techniques that have been considered for gene delivery of shRNA expression vectors include cationic lipids and liposomes, viruses and physical methods. However, a number of aspects limit the applicability of these methods in humans. The use of a viral vector has been developed as a highly efficient method for gene delivery to a variety of tissues, although it evokes specific immune responses that may limit clinical application. Among non-viral techniques, ultrasound-targeted microbubble destruction (UTMD) has evolved as a new, promising tool for site-specific drug and gene delivery in vitro and in vivo, targeting delivery via a process called sonoporation, allowing for direct transfer into the cells (14-16). Significant efforts have been made to demonstrate the application of siRNA mediated by UTMD to block gene expression in vitro and in vivo (17-20). Wang et al (21) found that UTMD was capable of delivering survivin siRNA into SKOV-3 cells, which inhibited survivin expression and induced apoptosis. This technology provided a new promising approach for siRNA delivery in vitro. In our previous study (22), shRNA-survivin was added to HeLa cells followed by UTMD, and the downregulation of survivin expression was assessed. We suggested that this technique effectively inhibited the expression of the target gene and induced cell apoptosis. 
Moreover, in a previous in vitro experimental study (23), we attempted to solve an important problem arising from the application of the non-viral gene transfer system of UTMD (combination of ultrasound exposure and liposome microbubbles) and PEI, particularly in the transfection of shRNA targeting survivin. However, the UTMD technique for the delivery of shRNA in vitro had not yet been optimized, and such methods of apoptosis induction and the efficacy of using UTMD technique and shRNA expression vectors had not been studied.

In the present study, we investigated whether or not the different shRNAs targeting survivin were capable of being transfected by the UTMD technique. Notably, UTMD parameters for the delivery system of shRNA in vitro were optimized. Furthermore, we investigated the effects of gene inhibition and apoptosis induction, which was not performed previously. The results revealed that the optimal irradiation parameters obtained higher transfection efficiency and did not affect the integrity of plasmid DNA. UTMD mediated survivin gene mRNA and protein knockdown significantly, and caused marked cell apoptosis.

\section{Materials and methods}

Cell culture. Human cervical cancer cell lines (HeLa) were obtained from the American Type Culture Collection (ATCC) and cultured in Dulbecco's modified Eagle's medium (DMEM) supplemented with $10 \%$ heat-inactivated fetal bovine serum (Invitrogen Biotechnology, Shanghai, China). Cultures were grown at $37^{\circ} \mathrm{C}$ in a humidified atmosphere, containing $5 \% \mathrm{CO}_{2}$.

Construction of shRNA expression vectors targeting survivin. DNA template oligonucleotides corresponding to the human survivin gene (GenBank accession no. NM_001168) were designed and synthesized as in our previous study [23]: survivin-shRNA1 (sense, 5'-GATCCGGAACTGGCCCTT CTTGGAGTTCAAGAGACTCCAAGAAGGGCCAGTTCT TTTTTGGAAG-3'); survivin-shRNA2 (sense, 5'-GATCCG ACTGGACAAGAGAAAGAGCCTTCAAGAGAGGCTCTT TCTCTGTCCAGTTTTTTTGGAAG-3'); survivin-shRNA3 (sense, 5'-GATCCGGGACCACCGCATCTCTACATTCAA GAGATGTAGAGATGCGGTGGTCCTTTTTTGGAAG-3'). These double strand oligonucleotides were subcloned into a linearized U6 promoter-driven pSIREN-DNR-DsRed-Express vector (BD Biosciences Clontech, USA) at the BamHI and EcoRI sites. The selected reconstructed plasmids for transfection were extracted and purified using a Qiaquick kit (Qiagen, Crawley, UK). Those specific recombinant shRNA vectors were termed pSIREN/S1, pSIREN/S2 and pSIREN/S3, respectively. Similarly, a non-specific control vector was constructed (pSIREN/con). Transient transfections were performed using Lipofectamine ${ }^{\mathrm{TM}} 2000$ (Invitrogen Biotechnology, Shanghai, China), as described previously $(24,25)$.

Optimization of survivin-shRNA with the UTMD technique. Briefly, cells were harvested by $0.25 \%$ trypsin digestion after reaching 80-90\% confluence and were resuspended in DMEM media $\left(300 \mu \mathrm{l} /\right.$ well) at a concentration of $1 \times 10^{7}$ cell $/ \mathrm{ml}(26)$. SonoVue microbubbles (Bracco, Milan, Italy) were used to promote cavitation and were reconstituted in saline solution at a concentration of 25-30 particles/cell, and slowly added to the cell suspension prior to ultrasound exposure (25). The UTMD experiments were performed in an exposure tank and the transducer (Accusonic, Metron Medical Australia Pty., Ltd.) was held in a positioning device at the base of the tank, as previously described (27).

Experiments were divided into the groups: negative control group, Lipofectamine-transfected groups (pSIREN/ $\mathrm{CON}+\mathrm{L}, \mathrm{pSIREN} / \mathrm{S} 1+\mathrm{L}, \mathrm{pSIREN} / \mathrm{S} 2+\mathrm{L}, \mathrm{pSIREN} / \mathrm{S} 3+\mathrm{L}$ ) and UTMD-treated groups (pSIREN/S1+UTMD, pSIREN/ S2+UTMD, pSIREN/S3+UTMD).

The study was approved by the Ethics Committee of The Third Affiliated Hospital of Guangzhou Medical College, Guangzhou, China.

Optimization of the UTMD protocol. Plasmid (final concentration $25 \mu \mathrm{g} / \mathrm{ml}$ ) was added to the cell suspension prior to ultrasound exposure. The cells were exposed to various ultrasound intensities $\left(0.4,1.0,1.6\right.$ and $\left.2.2 \mathrm{~W} / \mathrm{cm}^{2}\right)$ for various durations (1 and $3 \mathrm{~min}$ ), with a 10 or $20 \%$ of duty cycle (DC) and frequency of $1 \mathrm{MHz}, \mathrm{n}=6$ for each parameter.

Analysis of transfection efficiency. The transfected cell expression of red fluorescence protein (RFP) was visualized by an inverse fluorescence microscope (Olympus IX71, Japan) after $48 \mathrm{~h}$ (28). The cells were then harvested, collected and resuspended in phosphate-buffered saline (PBS). Approximately $1 \times 10^{5}$ cells were obtained from each sample for transfection efficiency analysis, with a 488-nm wavelength excitation light and a $585 \pm 42 \mathrm{~nm}$ wavelength emission light to detect the red fluorescence using flow cytometry (FACSCalibur, BectonDickinson, USA). The transfection efficiency was assessed as the number of cells expressing RFP per total number of surviving cells.

Stability of plasmid DNA following ultrasound exposure. Plasmid DNA $(100 \mu \mathrm{l})$ or SonoVue/DNA complexes were added into 6 wells of 96-well culture plates, as described previously (15), and exposed to ultrasound irradiation $(0.4,1.0$, 1.6 and $2.2 \mathrm{~W} / \mathrm{cm}^{2}$; DC, 20\%; exposure time, $3 \mathrm{~min}$ ) with or without the addition of SonoVue microbubbles. Gel electrophoresis analysis was performed immediately after ultrasound irradiation.

Reverse transcription PCR (RT-PCR). Cells were harvested $48 \mathrm{~h}$ following transfection, and total RNA was extracted with TRIzol reagent (Invitrogen) according to the manufacturer's instructions. The RNA was reverse-transcribed into cDNA with a reverse transcription kit (Promega, Madison, WI, USA). Specific primers were designed on the basis of human cDNA sequences using Primer Premier 5.0 software (Premier Biosoft International, Palo Alto, CA, USA) and synthesized by Invitrogen. The sequences of the primers were: forward, 5'-ACCACCGCATCTCTACATTC-3'; reverse, 5'-AGTCTG GCTCGTTCTCAGTG-3'; and the predicted product was 113 bp. GAPDH was used as an internal standard and its mRNA was amplified with primers: forward,5'-GAAGGTGAA GGTCGGAGTC-3'; reverse, 5'-GAAGATGGTGATGGG ATTTC-3'; and the predicted product was $226 \mathrm{bp}$. PCR amplification of the sequences was performed and the products 

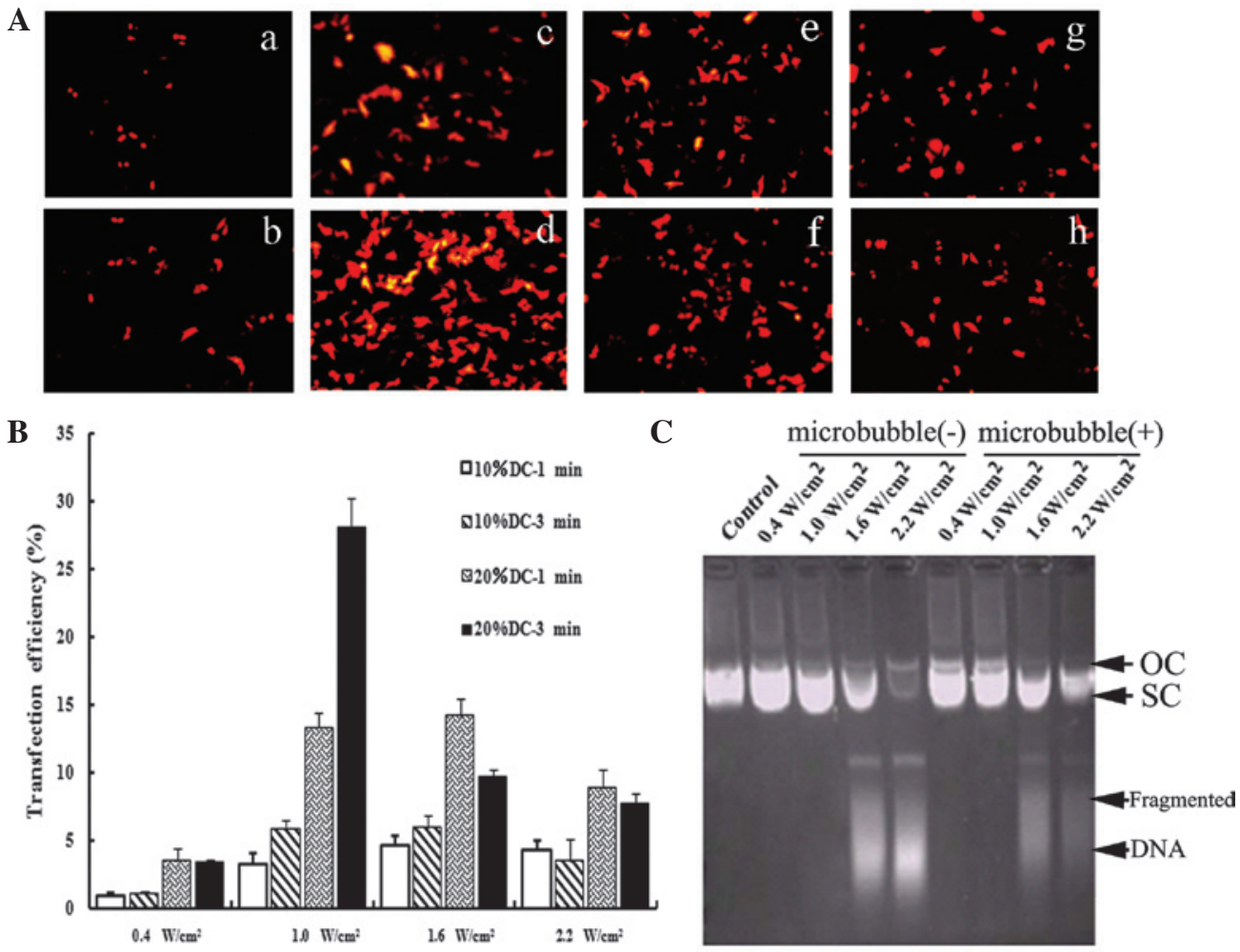

Figure 1. Optimization of the UTMD-mediated delivery system in vitro. (A) Fluorescence microscopy views under various ultrasound intensities $(0.4,1.0,1.6$ and $2.2 \mathrm{~W} / \mathrm{cm}^{2}$ ) and exposure times (1 and $3 \mathrm{~min}$ ), DC=20\%. With the optimal ultrasound parameters (Fig. la-d), the transfection efficiency was increased significantly $(28.04 \pm 2.27 \%)$. (B) Effects of ultrasound parameters on the transfection efficiency. When cells were irradiated using $10 \%$ DC with 0.4 W/cm ${ }^{2}$, only a few expressed RFP. When the ultrasound intensity was increased to $1.0 \mathrm{~W} / \mathrm{cm}^{2}$ or when the DC was $20 \%$, the transfection efficiency increased significantly (P<0.05). However, when the ultrasound intensity was further increased to 1.6 and $2.2 \mathrm{~W} / \mathrm{cm}^{2}$ or the exposure time was extended to 3 min, the expression of RFP decreased. As compared with $10 \%$ DC, irradiating for 1 and 3 min with $20 \%$ DC improved the expression rate significantly $(\mathrm{P}<0.05)$. $(\mathrm{C})$ Effects of ultrasound parameters on the integrity of plasmid DNA. Ultrasound parameters, $1.0 \mathrm{~W} / \mathrm{cm}^{2} ; \mathrm{DC}=20 \%$; exposure time, 3 min. The structures and migration rates of vectors did not markedly change following irradiation with 0.4 and $1.0 \mathrm{~W} / \mathrm{cm}^{2}$. Following treatment with higher ultrasound intensity, the structures of the vectors were markedly destroyed and DNA fragments could be observed. Under the same ultrasound parameters, the addition of SonoVue had no effect on the integrity of plasmid DNA. UTMD, ultrasound-targeted microbubble destruction; DC, duty cycle; RFP, red fluorescence protein; OC, open circular DNA; SC, supercoiled DNA.

obtained were electrophoresed through a $1 \%$ agarose gel with ethidium bromide. The intensity of survivin and GAPDH were evaluated using Quantity One software (Bio-Rad, Hercules, CA, USA). The relative expression levels of survivin were expressed as the ratio of survivin/GAPDH.

Western blotting. The cells were washed twice with cold PBS prior to being lysed in cell lysis buffer. Cell extracts were separated by $10 \%$ sodium dodecyl sulfate polyacrylamide gel electrophoresis (SDS-PAGE), and transferred onto nitrocellulose membranes (Millipore, Billerica, MA, USA). Immunoblot analysis was performed according to standard protocol (24). The blots were developed using enhanced chemiluminescence. The relative levels of survivin were expressed as the ratio of survivin $/ \beta$-actin.

DNA ladder formation. DNA was extracted from detached and adherent cells, and was subjected to electrophoresis using agarose gel as previously described (15).

Flow cytometry assays of apoptosis. Cells were assessed by flow cytometry (FACSCalibur, BectonDickinson) following the double staining of cells with annexin $\mathrm{V}$ and 7-AAD (Jinmei Biotech Co. Ltd., Wuhan, China). Early apoptotic cells were stained with FITC-labelled annexin-V alone, whereas necrotic and late apoptotic cells were stained with annexin $\mathrm{V}$ and 7-AAD (29). Each assay was performed three times.

Flow cytometric analysis of the cell cycle. A total of $48 \mathrm{~h}$ after transfection, HeLa cells were collected by trypsinization, pooled with non-attached cells, and fixed in $70 \%$ ethanol on ice for $30 \mathrm{~min}$. Fixed cells were pelleted by centrifugation and suspended in $10 \mathrm{mg} / \mathrm{ml}$ propidium iodide, $100 \mathrm{mg} / \mathrm{ml} \mathrm{RNase} \mathrm{A}$ and $0.05 \%$ Triton X-100 in PBS (pH 7.4), and finally stained with $20 \mathrm{mg} / \mathrm{ml}$ propidium iodide (PI; $300 \mathrm{ml}$ ) for $20 \mathrm{~min}$. Samples were analyzed for DNA content by flow cytometry. The samples were assayed in triplicate, and the fraction of every cell cycle phase was calculated.

Statistical analysis. Statistical analysis was performed using the SPSS 13.0 package (SPSS, Chicago, IL, USA). The variables were shown as the mean \pm standard deviation (SD). $\mathrm{P}<0.05$ was considered to indicate a statistically significant difference.

\section{Results}

Optimization of the UTMD-mediated delivery system in vitro. First, we investigated whether the UTMD technique was suitable for the delivery of shRNA into cells. As shown in Fig. 1A, 
A
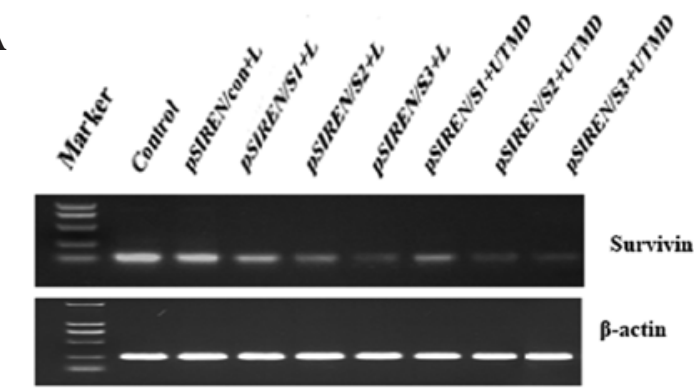

B
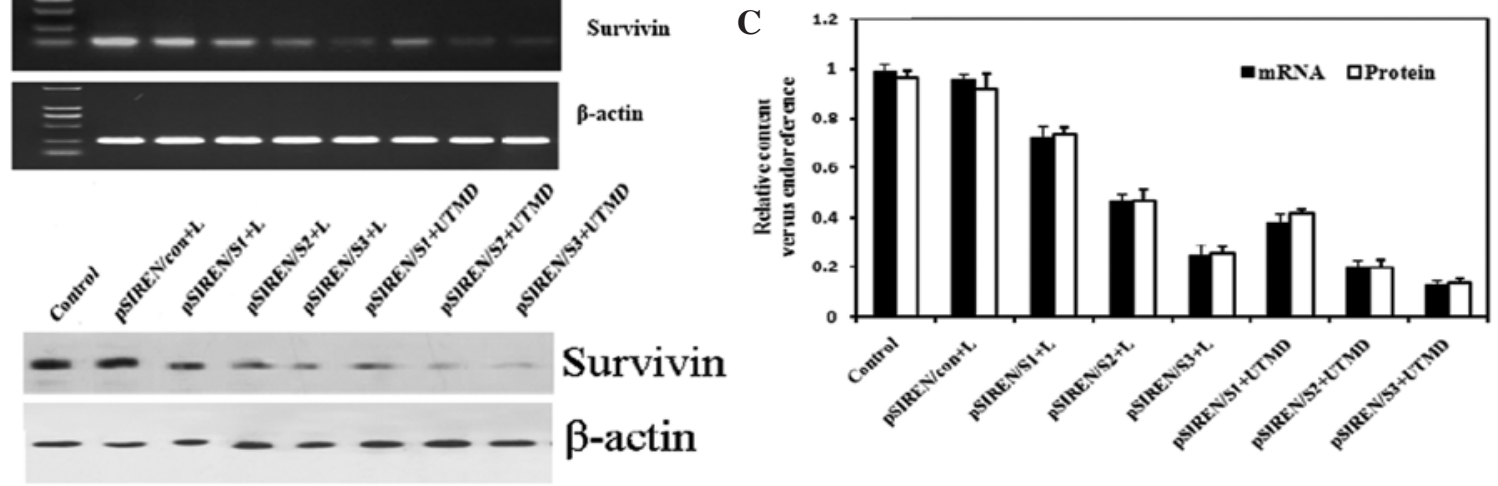

Figure 2. Vector-based shRNAs decreased endogenous survivin expression in cells. (A) Experiments in vitro were divided into 8 groups. The result of expression of survivin mRNA with semi-quantitative RT-PCR is shown. (B) Western blot of survivin expression in HeLa cell lines. (C) Experiments were performed $48 \mathrm{~h}$ after transfection of vector-based shRNAs in HeLa cells. The vectors mediated by the UTMD method had a better interfering effect on survivin mRNA and protein expression as compared with Lipofectamine-transfected samples. Moreover, the expression levels of survivin mRNA and protein were the lowest in the pSIREN/S3+UTMD group as compared with the other groups (P<0.05). UTMD, ultrasound-targeted microbubble destruction; M, marker, DL2000; L, Lipofectamine 2000 .

transfection efficiency was affected by transfection parameters, such as ultrasound intensity and exposure time. The optimal conditions of ultrasound exposure achieved the highest transfection efficiency (Fig. 1A-D). Moreover, our results showed that the appearance of DNA fragments was correlated with the transfection parameters (Fig. 1C). No significant effect was noted on the structure of vectors under suitable exposure conditions. The optimized parameters $\left(1.0 \mathrm{~W} / \mathrm{cm}^{2}\right.$ for 3 min with $20 \%$ DC pulse mode) were used in the subsequent UTMD-treated groups.

Vector-based shRNAs decreased endogenous survivin expression in cells. We examined whether delivery of survivin-shRNA by our UTMD system was capable of efficiently knocking down the expression of survivin. When shRNA expression vectors were introduced into HeLa cells, the results of RT-PCR showed that survivin mRNA expression was markedly knocked down, whereas the control or pSIREN/con groups did not have this effect (Fig. 2A and C). Furthermore, the expression levels of survivin mRNA declined markedly in the UTMD-treated groups as compared with the Lipofectaminetransfected groups $(\mathrm{P}<0.05$ for all). Western blot analysis also revealed that the expression of survivin protein was further suppressed in the groups transfected with $\mathrm{pSIREN} / \mathrm{S} 1$, pSIREN/S2 and pSIREN/S3 vectors (Fig. 2B and C). Transfection in the UTMD group was found to induce the greatest inhibition of survivin protein expression, as compared with the Lipofectamine-treated groups $(\mathrm{P}<0.05$ for all).

RNAi-targeting survivin inhibited apoptosis induction. To evaluate the effect of survivin depletion on the proliferation and apoptosis of HeLa cells in vitro, we used the flow cytometry assay to detect the effect of loss of survivin on cell apoptosis. The survivin shRNA construct-transfected HeLa cells showed a robust induction in cell apoptosis compared to the control
(Fig. 3). Our data demonstrated that the apoptosis ratio in the pSIREN/S3+UTMD group $(42.03 \pm 1.52 \%)$ was significantly higher than that of the pSIREN/S3+L group $(31.46 \pm 2.78 \%)$ (Fig. 3A, $\mathrm{P}<0.05$ ). To further determine the impact of survivin shRNA on cell growth, we detected the morphological changes in HeLa transfectants and the DNA ladder. The apoptotic cells in the pSIREN/S3+UTMD group presented typical morphological changes of cell apoptosis, such as cell shrinkage, round shape and bubbled cell membranes (Fig. 3B). Moreover, the apparent DNA ladder was detected in the pSIREN/S3+UTMD or pSIREN/S3+L groups, whereas there was no marked change in the control or non-specific vector groups (Fig. 3C).

Changes in the cell cycle apoptotic rate. Cell cycle distribution analysis by flow cytometry indicated that, as compared with the control group, there were marked changes in the $\mathrm{P}+\mathrm{L}$ and $\mathrm{P}+\mathrm{UTMD}$ groups; a number of cells were blocked in the $\mathrm{G}_{0} / \mathrm{G}_{1}$ phase $(70.81 \pm 1.78$ vs. $74.9 \pm 1.21$ vs. $56.54 \pm 2.01 \%, \mathrm{P}<0.01)$, reduced sharply in the $G_{2} / M$ phase $(6.11 \pm 0.67$ vs. $5.20 \pm 1.71$ vs. $19.89 \pm 1.77 \%, \mathrm{P}<0.01)$ and $\mathrm{S}$ phase $(23.08 \pm 1.29$ vs. $19.89 \pm 1.11$ vs. $23.56 \pm 1.99 \%, \mathrm{P}<0.01)$, and an apoptosis peak was observed prior to the $\mathrm{G}_{1}$ phase (Fig. $4 \mathrm{~A}$ ).

\section{Discussion}

Cervical carcinoma is a common high-risk malignancy in gynecology and its main therapeutic method is radiotherapy. Apoptosis induction is an effective and promising cancer treatment. Among the regulators of apoptosis, IAP proteins, such as survivin, have recently attracted considerable attention for their ability to suppress an evolutionarily conserved step in apoptosis, potentially involving direct caspase inhibition. Therefore, survivin has become a significant target for tumor therapy. In this study, we inhibited the expression of survivin to promote cell apoptosis and inhibit cell proliferation. 
A
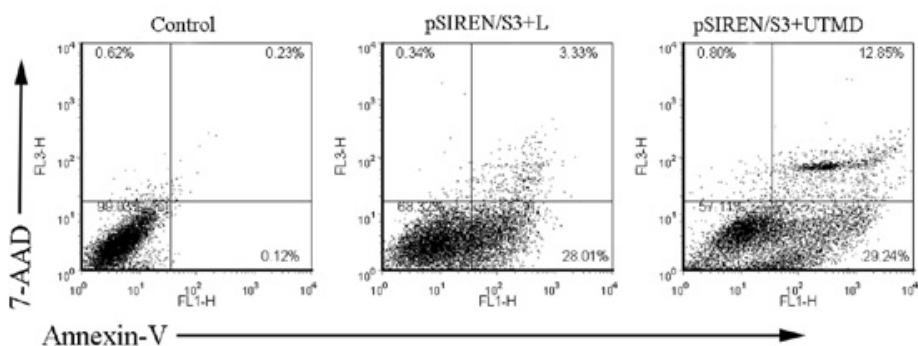

B

Control

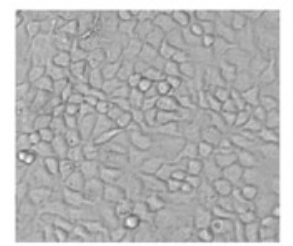

pSIREN/S3+UTMD
C

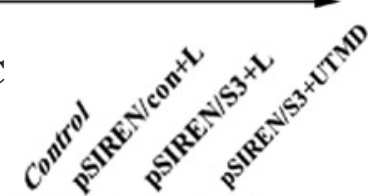

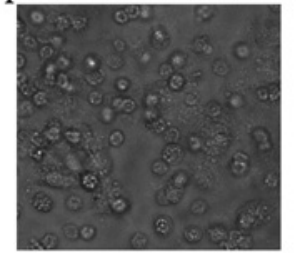

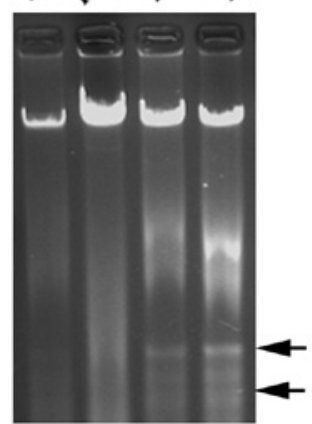

Figure 3. Apoptosis induction by shRNA targeting survivin with the UTMD technique in vitro. (A) Representative flow cytometric analysis of cell apoptosis FL1-H and FL3-H indicates the fluorescence intensity of FITC-annexin V and 7-AAD, respectively. (B) Morphological changes in HeLa transfectants were detected under a phase contrast microscope (original magnification, $\mathrm{x} 400$ ). The black arrows show the classical change of morphology in the UTMD group. Apparent apoptotic cells were observed in the UTMD-treated samples; original magnification, x200. (C) DNA ladder in different groups. Apoptosis was indicated by a ladder pattern (as shown by the arrows) in cells transfected with Lipofectamine or UTMD. UTMD, ultrasound-targeted microbubble destruction; shRNA, short hairpin RNA.

\section{A} $=\mathrm{GO}=\mathrm{G} 1 \mathrm{mS}$
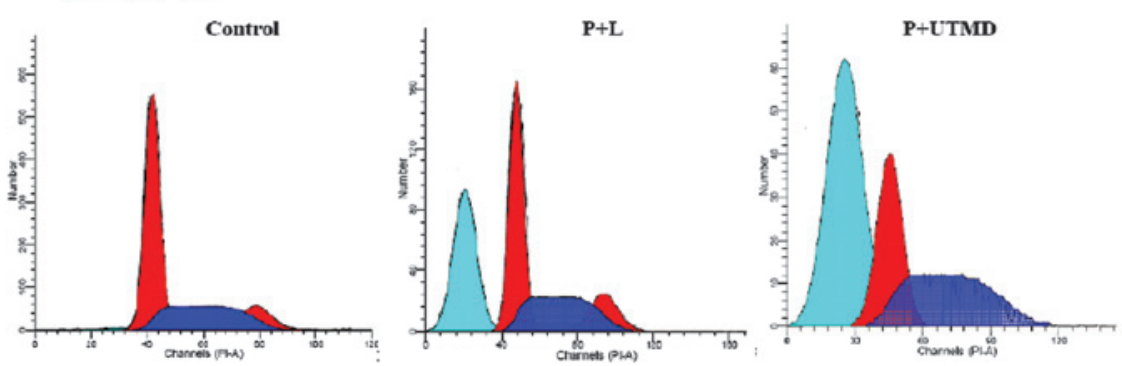

B

$\because \mathrm{G} 0 / \mathrm{G} 1 \| \mathrm{S} \quad \mathrm{G} 2 / \mathrm{M}$

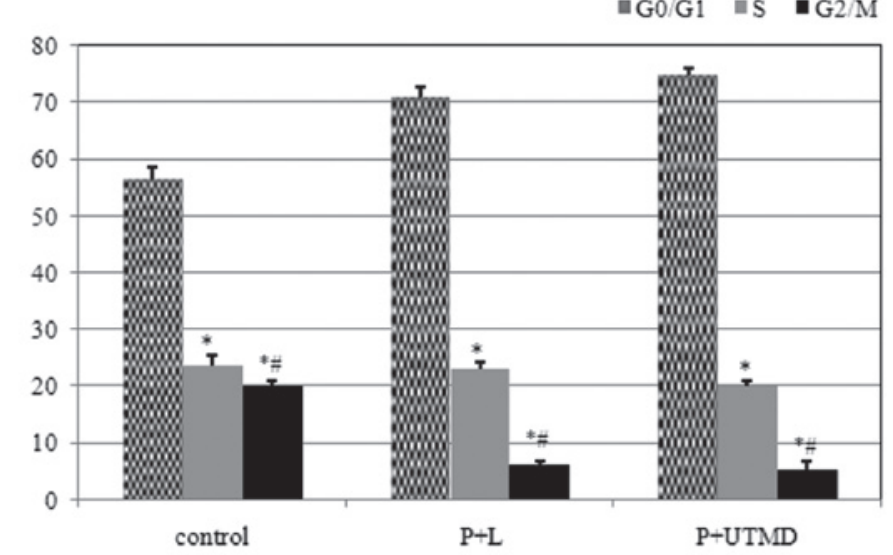

Figure 4. Comparison of cell cycle distribution by shRNA targeting of survivin with the UTMD technique in vitro. Data are the means \pm SE. Following a 48 -h culture, cells were analyzed for DNA content by propidium iodide staining and flow cytometry. Downregulation of survivin expression arrested the cell cycle in the $G_{0} / G_{1}$ period. Moreover, the percentage of the $P+L$ group and $P+U T M D$ group was markedly increased as compared with control groups $(P<0.01)$. Percentage of P+UTMD group was markedly increased as compared with $\mathrm{P}+\mathrm{L}$ group significantly $(\mathrm{P}<0.05)$. UTMD, ultrasound-targeted microbubble destruction; shRNA, short hairpin RNA; SE, standard error. 
In a previous study (22), pSIREN/S3 was added to HeLa cells followed by exposure to ultrasound and SonoVue microbubbles. The study was designed to evaluate the non-viral gene transfer system of UTMD compared with other transfection methods. We suggested that shRNA targeting the survivin gene mediated by the UTMD technique was capable of effectively inhibiting the expression of target genes and induce cell apoptosis. In a previous study (23), we hypothesized that three shRNAs targeting the survivin gene could be transfected by ultrasound with liposome microbubbles. In this study, we investigated whether the shRNA expression vectors targeting survivin (pSIREN/S1, pSIREN/S2, pSIREN/S3 and pSIREN/ CON) were capable of being transfected using the UTMD technique. Following transfection of the vectors into the human cervical cancer cells, we measured their inhibitory effects on survivin mRNA and protein expression by semi-quantitative RT-PCR and western blotting. Transfection efficiency with flow cytometry assays and the stability of plasmid DNA was detected. The results indicated that shRNA targeting survivin mediated by the UTMD technique markedly inhibited the expression of survivin mRNA and its corresponding protein to a certain degree in vitro. Moreover, $\mathrm{pSIREN/S3}$ has a higher inhibitory power not only in the Lipofectamine-transfected group but also in the UTMD-treated group, and the pSIREN/ S3 vector combined with the UTMD method had a better interfering effect on survivin expression in the HeLa cells, and was selected for further analysis. The novel data reported in this study included the combination of ultrasound exposure and SonoVue microbubbles and optimization of the UTMD technique for the delivery system of shRNA in vitro, which has not been performed in previous studies.

The results of our study and other reports $(16,30)$ have shown that, prior to achieving the optimal parameters, such as increasing ultrasound intensity, extending irradiation time or DC, UTMD may improve transfection efficiency. However, when plasmid DNA was treated with high intensity or low frequency ultrasound, shear stress mediated by UTMD led to the structural degradation of plasmid DNA (31). Certain measures should be taken to protect vectors, to further enhance the transfection efficiency. Our results reveal that UTMD-mediated survivin gene mRNA and protein knockdown in HeLa cell lines caused marked cell apoptosis and that the appearance of DNA fragments was correlated with the transfection parameters. The optimal irradiation parameters would achieved higher transfection efficiency, but exhibited no effect on the integrity of plasmid DNA.

We hypothesized that the downregulation of survivin was capable of inducing apoptosis in cancer cells. To explore this possibility, we measured levels of apoptosis in transfected and control cells. Experimental data indicated that degradation of survivin was the main trigger for apoptosis induction. After the expression of survivin was downregulated, a marked block in the $G_{0} / G_{1}$ phase of the cell cycle was observed and cell apoptosis induction was examined, which was similar to other reports that did not use this technique (32). These data have laid a foundation for further investigation into cervical carcinoma therapeutics.

In conclusion, our results showed that survivin downregulation with shRNA expression vectors mediated by the optimal UTMD parameters markedly induced cell apoptosis and caused cell cycle arrest. This study suggests that a combination treatment may lead to an additive apoptosis-inducing effect on cervical cancer cells. More studies are required to promote this efficient, promising novel technique for gene delivery, which is likely to accelerate the development of therapeutic approaches for human cervical cancer.

\section{Acknowledgements}

This study was supported by the Medical Research Foundation of Guangdong Province (No. A2010270), and by research projects of the Guangzhou Education Bureau (No. 10A242), and by research projects of the Science and Technology Bureau of Guangzhou Liwan District (20111213067), and by youth research support projects of the Third Affiliated Hospital of Guangzhou Medical University (2011Y02).

\section{References}

1. Evan GI and Vousden KH: Proliferation, cell cycle and apoptosis in cancer. Nature 411: 342-348, 2001.

2. Lakhani SA, Masud A, Kuida K, Porter GA Jr, Booth CJ, Mehal WZ, Inayat I and Flavell RA: Caspases 3 and 7: key mediators of mitochondrial events of apoptosis. Science 311: 847-851, 2006.

3. Chipuk JE, Kuwana T, Bouchier-Hayes L, Droin NM, Newmeyer DD, Schuler M and Green DR: Direct activation of Bax by p53 mediates mitochondrial membrane permeabilization and apoptosis. Science 303: 1010-1014, 2004.

4. Knauer SK, Krämer OH, Knösel T, Engels K, Rödel F, Kovács AF, Dietmaier W, Klein-Hitpass L, Habtemichael N, Schweitzer A, Brieger J, Rödel C, Mann W, Petersen I, Heinzel T and Stauber RH: Nuclear export is essential for the tumorpromoting activity of survivin. FASEB J 21: 207-216, 2007.

5. Carvalho A, Carmena M, Sambade C, Earnshaw WC and Wheatley SP: Survivin is required for stable checkpoint activation in taxol-treated HeLa cells. J Cell Sci 116: 2987-2998, 2003.

6. Caldas H, Holloway MP, Hall BM, Qualman SJ and Altura RA: Survivin-directed RNA interference cocktail is a potent suppressor of tumour growth in vivo. J Med Genet 43: 119-128, 2006.

7. Kappler M, Bache M, Bartel F, Kotzsch M, Panian M, Würl P, Blümke K, Schmidt H, Meye A and Taubert H: Knockdown of survivin expression by small interfering RNA reduces the clonogenic survival of human sarcoma cell lines independently of p53. Cancer Gene Ther 11: 186-193, 2004.

8. Liu Q, Fu H, Xing R, Tie Y, Zhu J, Sun Z and Zheng X: Survivin knockdown combined with apoptin overexpression inhibits cell growth significantly. Cancer Biol Ther 7: 1053-1060, 2008.

9. Vlassov AV, Korba B, Farrar K, Mukerjee S, Seyhan AA, Ilves H, Kaspar RL, Leake D, Kazakov SA and Johnston BH: shRNAs targeting hepatitis $\mathrm{C}$ : effects of sequence and structural features, and comparision with siRNA. Oligonucleotides 17: 223-236, 2007.

10. Sui G, Soohoo C, Affar el B, Gay F, Shi Y, Forrester WC and Shi Y: A DNA vector-based RNAi technology to suppress gene expression in mammalian cells. Proc Natl Acad Sci USA 99: 5515-5520, 2002.

11. McAnuff MA, Rettig GR and Rice KG: Potency of siRNA versus shRNA mediated knockdown in vivo. J Pharm Sci 96: 2922-2930, 2007.

12. Boudreau RL, Monteys AM and Davidson BL: Minimizing variables among hairpin-based RNAi vectors reveals the potency of shRNAs. RNA 14: 1834-1844, 2008.

13. Pardridge WM: shRNA and siRNA delivery to the brain. Adv Drug Deliv Rev 59: 141-152, 2007.

14. Guo DP, Li XY, Sun P, Tang YB, Chen XY, Chen Q, Fan LM, Zang B, Shao LZ and Li XR: Utrasound-targeted microbubble destruction improves the low density lipoprotein receptor gene expression in HepG2 cells. Biochem Biophys Res Commun 343: 470-474, 2006.

15. Chen ZY, Xie MX, Wang XF, Lü Q and Ding SW: Efficient gene delivery to myocardium with ultrasound targeted microbubble destruction and polyethylenimine. J Huazhong Univ Sci Technolog Med Sci 28: 613-617, 2008. 
16. Chen ZY, Xie MX, Wang XF and Lü Q: Effects of lipid shell microbubble on ultrasound mediated EGFP gene delivery to transplanted tumors: initial experience. Chin-Ger J Clin Oncol 7: 424-428, 2008.

17. Kinoshita $\mathrm{M}$ and Hynynen $\mathrm{K}$ : A novel method for the intracellular delivery of siRNA using microbubble-enhanced focused ultrasound. Biochem Biophys Res Commun 335: 393-399, 2005.

18. Saito M, Mazda O, Takahashi KA, Arai Y, Kishida T, Shin-Ya M, Inoue A, Tonomura H, Sakao K, Morihara T, Imanishi J, Kawata $M$ and Kubo T: Sonoporation mediated transduction of pDNA/siRNA into joint synovium in vivo. J Orthop Res 25: 1308-1316, 2007.

19. Tran MA, Gowda R, Sharma A, Park EJ, Adair J, Kester M, Smith NB and Robertson GP: Targeting V600EB-Raf and Akt3 using nanoliposomal-small interfering RNA inhibits cutaneous melanocytic lesion development. Cancer Res 68: 7638-7649, 2008.

20. Vandenbroucke RE, Lentacker I, Demeester J, De Smedt SC and Sanders NN: Ultrasound assisted siRNA delivery using PEG-siPlex loaded microbubbles. J Control Release 126 265-273, 2008.

21. Wang J, Zheng Y, Yang F, Zhao P and Li H: Survivin small interfering RNA transfected with a microbubble and ultrasound exposure inducing apoptosis in ovarian carcinoma cells. Int J Gynecol Cancer 20: 500-506, 2010.

22. Chen ZY, Liang K, Xie MX, Wang XF, Lü Q and Zhang J: Novel ultrasound-targeted microbubble destruction mediated short hairpin RNA plasmid transfection targeting survivin inhibits gene expression and induces apoptosis of HeLa cells. Mol Biol Rep 36: 2059-2067, 2009.

23. Chen ZY, Liang K, Liu JH, Xie MX, Wang XF, Lü Q, Zhang J and Fang LY: Enhancement of survivin gene downregulation and cell apoptosis by a novel combination: liposome microbubbles and ultrasound exposure. Med Oncol 26: 491-500, 2009.
24. Ling $X$ and Li F: Silencing of antiapoptotic survivin gene by multiple approaches of RNA interference technology. Biotechniques 36: 450-454, 456-460, 2004.

25. Mehier-Humbert S, Bettinger T, Yan F and Guy RH: Ultrasoundmediated gene delivery: kinetics of plasmid internalization and gene expression. J Control Release 104: 203-211, 2005.

26. Zarnitsyn VG and Prausnitz MR: Physical parameters influencing optimization of ultrasound-mediated DNA transfection. Ultrasound Med Biol 30: 527-538, 2004.

27. Kodama T, Tomita Y,Koshiyama K and Blomley MJ: Transfection effect of microbubbles on cells in superposed ultrasound waves and behavior of cavitation bubble. Ultrasound Med Biol 32: 905-914, 2006.

28. Li H, Wang S, Zhu T, Zhou J, Xu Q, Lu Y and Ma D: Pin1 contributes to cervical tumorigenesis by regulating cyclin D1 expression. Oncol Rep 16: 491-496, 2006.

29. Ochiai N, Uchida R, Fuchida S, Okano A, Okamoto M, Ashihara E, Inaba T, Fujita N, Matsubara $\mathrm{H}$ and Shimazaki C: Effect of farnesyl transferase inhibitor R115777 on the growth of fresh and cloned myeloma cells in vitro. Blood 102: 3349-3353, 2003.

30. Liang HD, Lu QL, Xue SA, Halliwell M, Kodama T, Cosgrove DO, Stauss HJ, Partridge TA and Blomley MJ: Optimisation of ultrasound-mediated gene transfer (sonoporation) in skeletal muscle cells. Ultrasound Med Biol 30: 1523-1529, 2004.

31. Kuo JH, Jan MS and Sung KC: Evaluation of the stability of polymer-based plasmid DNA delivery systems after ultrasound exposure. Int J Pharm 257: 75-84, 2003.

32. Zhang R, Ma L, Zheng M, Ren J, Wang T, Meng Y, Zhao J, Jia L, Yao L, Han H, Li K and Yang A: Survivin knockdown by short hairpin RNA abrogates the growth of human hepatocellular carcinoma xenografts in nude mice. Cancer Gene Ther 17: 275-288, 2010 